\title{
Edge magnetoplasmons and the optical excitations in graphene disks
}

\author{
Weihua Wang, ${ }^{*}$ S. Peter Apell, and Jari M. Kinaret \\ Department of Applied Physics, Chalmers University of Technology, SE-412 96 Göteborg, Sweden \\ (Received 5 July 2012; revised manuscript received 10 September 2012; published 28 September 2012)
}

\begin{abstract}
We discuss the edge magnetoplasmon properties in highly doped graphene disks, and the corresponding optical excitations. Edge magnetoplasmons with nonzero angular momentum $(l \neq 0)$ have two branches corresponding to different edge current rotations with respect to the magnetic field. The resonance energies of one branch are blueshifted and the other redshifted relative to energies at $B=0$, with the energy differences linearly proportional to the magnetic field. Recently, the $l=1$ dipole mode has been investigated by two experiments using optical transmission spectroscopy [Crassee et al., Nano Lett. 12, 2470 (2012); Yan et al., ibid. 12, 3766 (2012)], and classical cyclotron resonances were found in highly doped graphene samples. These are determined by graphene magneto-optical conductivities, which behave like a conventional two-dimensional electron system in the high doping limit.
\end{abstract}

DOI: 10.1103/PhysRevB.86.125450

PACS number(s): 73.20.Mf, 71.35.Ji, 78.67.Wj

\section{INTRODUCTION}

Plasmons are fundamental collective oscillations of electrons that have captured the interest of scientists for years, driving research in theory, experiment, and applications. Dirac plasmons of graphene, ${ }^{1}$ which have also been identified recently, are predicted to have particularly interesting and unique properties, e.g., longer plasmon propagating length and higher field confinement. As an ideal two-dimensional electron system (2DES), the carrier concentration in graphene can be controlled through chemical doping or electrostatic gating. ${ }^{2-5}$ Hence, one can tailor the local conductivity by a bias voltage, and it might provide exciting venues to achieve wave-guiding in simple graphene flakes without additional nanostructures, which have been suggested to perform transformation optics and cloaking on a one-atom-thick surface. ${ }^{6,7}$ Engineering plasmonic nanostructures in graphene, such as disks and ribbons, enables rich functionalities to be obtained, such as modulating the emitter radiation, ${ }^{8}$ enhancing the light matter interaction, and realizing plasmon wave-guiding. ${ }^{9,10}$ These kinds of structures have also been demonstrated theoretically to achieve a very high optical absorption. ${ }^{11,12}$ Quantum effects are also very important in small-sized graphene nanostructures, ${ }^{13}$ and very recently, atomically localized plasmon was observed in a graphene flake with a sub-nanoscale localized defect. ${ }^{14}$ From microsize down to nanosize, graphene plasmonics has the potential to bridge the size gap between electronics and photonics, ${ }^{15,16}$ and it may enable new functional optoelectronics devices to be implemented.

\section{MAGNETO-OPTICAL CONDUCTIVITIES OF GRAPHENE}

Active tunability is a key issue in plasmonics. In metalbased plasmonics, the designed nanostructures can usually only work at a specific frequency. However, in graphene plasmonics, the electron concentration can be easily tuned by electrostatic gating, so the plasmon frequency will be tuned as well (e.g., the dipolar plasmon frequencies depend on the chemical potential ${ }^{11}$ ). Very recently, this idea was demonstrated experimentally by two groups using a scatteringtype scanning near-field optical microscope. ${ }^{17,18}$ In addition to this electric tuning method, graphene plasmons can also be tuned through magnetic fields. Graphene magnetoplasmons have been studied theoretically in infinite graphene sheets, ${ }^{19-22}$ semi-infinite sheets, ${ }^{23,24}$ and finite structures. ${ }^{25,26}$ As a linear and gapless energy spectrum, graphene has a different Landau level (LL) distribution compared with a usual 2DES, reading

$$
E_{n}= \pm \sqrt{n} \hbar \omega_{B},
$$

where $\omega_{B}=\sqrt{2} v_{F} / l_{B}$, and the \pm represent an electronlike $(+)$ or holelike $(-)$ LL index. Here $v_{F} \approx 10^{6} \mathrm{~m} / \mathrm{s}$ is the Fermi velocity in graphene and $l_{B}=\sqrt{\hbar / e B}$ is the magnetic length. With this energy dispersion, the conductivities can be calculated using the Kubo formula, ${ }^{27}$

$$
\begin{aligned}
\sigma_{x x}(\omega)= & \frac{e^{2}}{2 \pi \hbar} \sum_{n=0}^{\infty}\left\{i \omega_{B}\left(\omega+i \tau^{-1}\right) \frac{\left[n_{F}\left(E_{n}\right)-n_{F}\left(E_{n+1}\right)\right]+\left[n_{F}\left(-E_{n+1}\right)-n_{F}\left(-E_{n}\right)\right]}{\left[\left(\omega+i \tau^{-1}\right)^{2}-f_{\text {intra }}^{2}(n) \omega_{B}^{2}\right] f_{\text {intra }}(n)}\right. \\
& \left.+i \omega_{B}\left(\omega+i \tau^{-1}\right) \frac{\left[n_{F}\left(-E_{n}\right)-n_{F}\left(E_{n+1}\right)\right]+\left[n_{F}\left(-E_{n+1}\right)-n_{F}\left(E_{n}\right)\right]}{\left[\left(\omega+i \tau^{-1}\right)^{2}-f_{\text {inter }}^{2}(n) \omega_{B}^{2}\right] f_{\text {inter }}(n)}\right\}, \\
\sigma_{x y}(\omega)= & \frac{e^{2}}{2 \pi \hbar} \sum_{n=0}^{\infty}\left\{\left[n_{F}\left(E_{n}\right)-n_{F}\left(E_{n+1}\right)\right]-\left[n_{F}\left(-E_{n+1}\right)-n_{F}\left(-E_{n}\right)\right]\right\} \\
& \times\left\{\frac{\omega_{B}^{2}}{\left(\omega+i \tau^{-1}\right)^{2}-f_{\text {intra }}^{2}(n) \omega_{B}^{2}}+\frac{\omega_{B}^{2}}{\left(\omega+i \tau^{-1}\right)^{2}-f_{\text {inter }}^{2}(n) \omega_{B}^{2}}\right\},
\end{aligned}
$$


where $n_{F}\left(E_{n}\right)=1 /\left\{1+\exp \left[\left(E_{n}-\mu_{c}\right) / k_{B} T\right]\right\}$ is the FermiDirac distribution, $\mu_{c}$ is the chemical potential, and

$$
\begin{aligned}
& f_{\text {intra }}(n)=\sqrt{n+1}-\sqrt{n}, \\
& f_{\text {inter }}(n)=\sqrt{n+1}+\sqrt{n} .
\end{aligned}
$$

These two functions determine the energy spectra of intraband and interband single-particle transitions. In the following quasistatic calculations and full wave simulations, we use a low temperature, $T=4.2 \mathrm{~K}$, and an intrinsic relaxation time, $\tau=\mu \mu_{c} / e v_{F}^{2},{ }^{9}$ where mobility $\mu=10^{4} \mathrm{~cm}^{2} / \mathrm{V} \mathrm{s}$.

\section{EDGE MAGNETOPLASMONS IN GRAPHENE DISKS}

In the long-wavelength limit, using the quasistatic approximation, the edge magnetoplasmon dispersions in disks of radius $R$ can be obtained by solving an integral equation,

$$
\Phi(r)=\frac{1}{\epsilon} \int_{0}^{R} d r^{\prime} K_{l}\left(r, r^{\prime}\right) r^{\prime}\left[\rho\left(r^{\prime}\right)+\rho^{*}\left(r^{\prime}\right)\right],
$$

with the integral kernel

$$
K_{l}\left(r, r^{\prime}\right)=\frac{1}{2} \int_{0}^{\infty} d p J_{l}(p r) J_{l}\left(p r^{\prime}\right),
$$

where $l$ is the angular momentum and $J_{l}$ is the Bessel function of first kind. The corresponding two-dimensional (2D) sheet and edge charge densities are

$$
\begin{aligned}
\rho(r) & =\frac{\sigma_{r r}}{i \omega}\left[\frac{l^{2}}{r^{2}}-\frac{1}{r} \frac{\partial}{\partial r} r \frac{\partial}{\partial r}\right] \Phi(r), \\
\rho^{*}(r) & =\frac{\delta(r-R)}{i \omega}\left[\sigma_{r r} \frac{\partial}{\partial r} \pm \sigma_{r \theta} \frac{i l}{r}\right] \Phi(r) .
\end{aligned}
$$

Here $\delta(r-R)$ arises from the step function in the conductivity tensor at the edge, and \pm correspond to different edge current rotations. Using a polynomial expansion technique, ${ }^{28,29}$ we can transfer the integral equation (4) into a standard eigenvalue matrix equation. Defining a dimensionless parameter $x=r / R$, we expand the potential as

$$
\Phi(x)=\sum_{j=0}^{\infty} c_{j} x^{l} P_{j}^{(l, 0)}\left(1-2 x^{2}\right) .
$$

Taking into account the orthogonality of Jacobi polynomials, ${ }^{28,29}$

$$
\int_{0}^{1} d x x^{2 l+1} P_{i}^{(l, 0)}\left(1-2 x^{2}\right) P_{j}^{(l, 0)}\left(1-2 x^{2}\right)=\frac{\delta_{i j}}{2(l+2 j+1)},
$$

multiplying by $x^{l+1} P_{i}^{(l, 0)}\left(1-2 x^{2}\right)$, and integrating Eq. (4), we obtain

$$
\frac{c_{i}}{2(l+2 i+1)}=\sum_{j=0}^{\infty}\left[\eta M_{i j}+\eta N_{i j} \pm \chi O_{i j}\right] c_{j} .
$$

The parameters which contain all material properties are defined as

$$
\begin{aligned}
& \eta=\frac{\sigma_{r r}}{i \omega \epsilon R}, \\
& \chi=\frac{\sigma_{r \theta}}{\omega \epsilon R},
\end{aligned}
$$
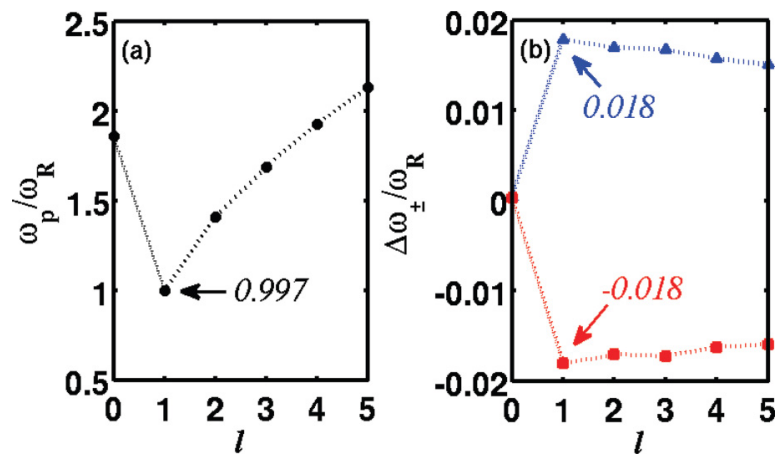

FIG. 1. (Color online) In the quasistatic approximation (a) Dispersion relations in the absence of magnetic field for a highly doped graphene disk. The energy of the optical active mode $(l=1)$ is $0.997 \omega_{R}$. The axisymmetric mode $(l=0)$ is a bulk resonance with energy $\omega=1.86 \omega_{R}$; (b) two edge magnetoplasmon excitations for $\mu_{c}=0.5 \mathrm{eV}, R=50 \mathrm{~nm}$, and $B=5 \mathrm{~T}$. Here $\Delta \omega_{ \pm}=\omega_{ \pm}-\omega_{p}$. For the $l=0$ mode, both are slightly blueshifted, while for the $l \neq 0$ modes, $\omega_{+}$is blueshifted and $\omega_{-}$is redshifted.

and the matrix elements are given by

$$
\begin{aligned}
M_{i j}= & \int_{0}^{1} d x x^{l+1} P_{i}^{(l, 0)} \int_{0}^{1} d x^{\prime} K_{l}\left(x, x^{\prime}\right) x^{\prime} \\
& \times\left[\frac{l^{2}}{x^{\prime 2}}-\frac{1}{x^{\prime}} \frac{\partial}{\partial x^{\prime}} x^{\prime} \frac{\partial}{\partial x^{\prime}}\right] x^{\prime l} P_{j}^{(l, 0)}, \\
N_{i j}= & \int_{0}^{1} d x x^{l+1} P_{i}^{(l, 0)} K_{l}(x, 1) \Phi^{\prime}\left(x^{\prime}\right)_{x^{\prime}=1}, \\
O_{i j}= & l \int_{0}^{1} d x x^{l+1} P_{i}^{(l, 0)} K_{l}(x, 1) \Phi\left(x^{\prime}\right)_{x^{\prime}=1} .
\end{aligned}
$$

In numerical calculations, enough polynomial terms in Eq. (7) are used to obtain convergent results; for instance, $j$ up to 14 indicating 15 terms gives a good precision. The numerical results are shown in Fig. 1. In the absence of the magnetic field, the plasmon energies for angular momenta $l$ up to 5 are shown, and the lowest plasmon energy happens at $l=1$, given by $\omega_{p}=0.997 \omega_{R}$, where $\omega_{R}^{2}=\mu_{c} e^{2}\left(2 \pi \hbar^{2} \epsilon R\right)^{-1}$. Here, $\epsilon$ is the surrounding dielectric constant, defined as the average dielectric constant of above and below the graphene layer, e.g., in a free-standing situation, $\epsilon=\epsilon_{0}\left(\epsilon_{r 1}+\epsilon_{r 2}\right) / 2=\epsilon_{0}$. In contrast to $2 \mathrm{D}$ plasmon energy in an infinite graphene sheet in the limit of long wavelength, ${ }^{30,31} \omega_{q}^{2}=\mu_{c} e^{2} q\left(2 \pi \hbar^{2} \epsilon\right)^{-1}$, we found that dipole resonances have approximately 2D plasmon energy at $q=R^{-1}$ (at dipole resonances, $l=q R=1$, which also indicates $q=R^{-1}$ ). The plasmons discussed here are the long-wavelength classical plasmons, and thus they are different from the atomic quantum plasmons which should be excited by high energy and large momentum transfer. ${ }^{32,33}$

We now compare the quasistatic results discussed above with a numerical solution of Maxwell's equations using finite-element method (FEM) commercial software COMSOL 4.0a. We model graphene disks as three-dimensional (3D) disks of very small thickness $t$, and the dielectric function $\epsilon_{g}=\epsilon+i \sigma / \omega t,{ }^{9}$ where $\sigma$ is the graphene conductivities given by Eqs. (2a) and (2b), and similarly $\epsilon$ is the surrounding dielectric constant. For example, in a perpendicular magnetic 

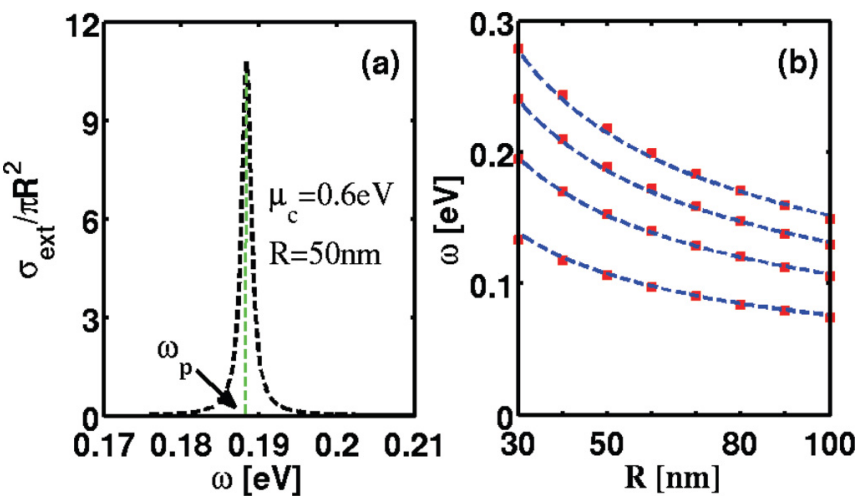

FIG. 2. (Color online) (a) Optical extinction spectrum obtained from FEM simulation. The peak indicates the dipole plasmon resonance; (b) extracted plasmon energies (red square dots) from FEM simulations, compared to $0.997 \omega_{R}$ (blue dashed lines). The four curves are for different chemical potentials: 0.2, 0.4, 0.6, and $0.8 \mathrm{eV}$ (from bottom to top).

field, the dielectric function tensor can be written as

$$
\epsilon_{g}=\epsilon+\frac{i}{\omega t}\left[\begin{array}{lcc}
\sigma_{x x} & \sigma_{x y} & 0 \\
-\sigma_{x y} & \sigma_{x x} & 0 \\
0 & 0 & \sigma
\end{array}\right]
$$

where $\sigma=\left.\sigma_{x x}\right|_{B=0}$, and in the absence of the magnetic field $\left(\sigma_{x y}=0\right)$, the dielectric function is isotropic. Basically, the method requires the radius $R$ to be much larger than the thickness $t$, and $t=0.5 \mathrm{~nm}$ can establish convergence of our simulations. ${ }^{9}$ Figure 2 (a) shows an extinction spectrum of a free-standing graphene disk, where the plane wave is normally incident with polarization in the disk plane. The spectral peak position corresponds to dipole plasmon energy. In Fig. 2(b), the plasmon energies extracted from FEM simulations and estimated using $\omega_{p}=0.997 \omega_{R}$ show good agreement for different chemical potentials and different disk radii. The plasmon resonance energies in graphene disks have also been investigated in Ref. 11 using the boundary element method (BEM), where the results for the dipole mode are also approximately predicted by $\omega_{p} \approx \omega_{R}$. We can conclude that the dipole plasmon energies in highly doped graphene disks are roughly equal to the $2 \mathrm{D}$ plasmon energies at $q=R^{-1}$.

In the presence of a magnetic field, there are two kinds of magnetoplasmons with different edge current rotations. ${ }^{28,34}$ We calculated the magnetoplasmon energies $\left(\omega_{ \pm}\right)$for different angular momenta $l$ from Eq. (9), and we plotted $\Delta \omega_{ \pm}=$ $\omega_{ \pm}-\omega_{p}$ in Fig. 1(b). For $l=0$ axisymmetric bulk mode, the magnetoplasmon energies are slightly blueshifted, while for $l \neq 0$ modes, the two magnetoplasmons are oppositely shifted and nearly symmetric with respect to no magnetic field, e.g., for $l=1$ the shifts are $\pm 0.018 \omega_{R}$.

We also studied the magnetoplasmon properties using FEM simulations. The magenta line (circles) shown in Fig. 3(a) is the extinction spectrum of a free-standing graphene disk in a magnetic field. There are two spectral peaks, corresponding
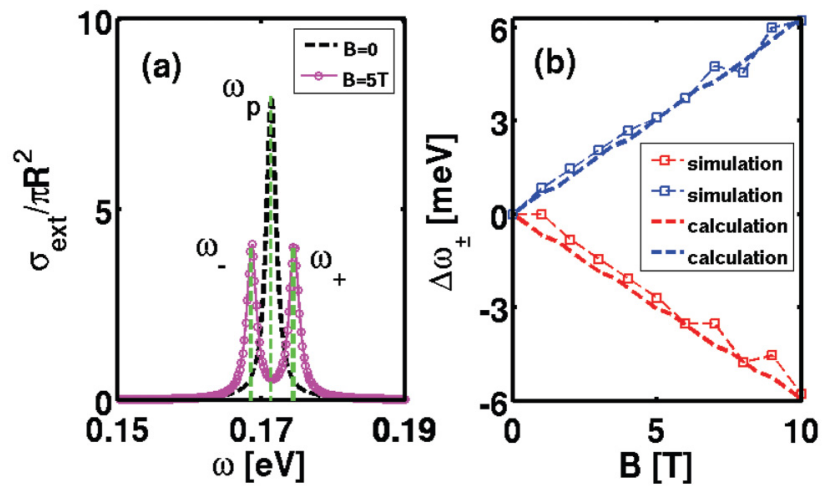

FIG. 3. (Color online) (a) Magnetoplasmon extinction spectrum (magenta circle line) simulated using COMSOL for $B=5 \mathrm{~T}, \mu_{c}=0.5$ $\mathrm{eV}$, and $R=50 \mathrm{~nm}$. Black dashed line represents plasmon excitation at $B=0$; (b) $\Delta \omega_{ \pm}=\omega_{ \pm}-\omega_{p}$, extracted from COMSOL simulations (square dashed lines) and calculated in the quasistatic approximation for the $l=1$ mode (dashed lines), show good agreement and exhibit an approximately linear dependence on $B$.

to two magnetoplasmon modes as discussed above. In the simulations, a linearly polarized plane wave is used, $\vec{E}=$ $\hat{e}_{x} E_{0} \exp [i(k z-\omega t)]$, which contains two circularly polarized components, $\hat{e}_{x}=\left(\hat{e}_{x}+i \hat{e}_{y}\right) / 2+\left(\hat{e}_{x}-i \hat{e}_{y}\right) / 2$. We found that only one magnetoplasmon resonance peak in Fig. 3(a) can be obtained for circularly polarized waves incidence. It is consistent with Eq. (6b) that different circularly polarized waves can couple to the respective edge current rotations. This could pave the way to study each magnetoplasmon mode separately in the experiments.

In Fig. 3(b), we also see that $\Delta \omega_{ \pm}$depends almost linearly on magnetic field $B$, revealed by both quasistatic calculations and FEM simulations. In graphene, the cyclotron energies given by Eq. (1) are $\propto \sqrt{B}$; however, in highly doped graphene systems, the cyclotron resonances exhibit classical behavior, $\omega_{c} \propto B$. This phenomenon has been demonstrated by Witowski and coauthors, ${ }^{35}$ and it was also observed very recently in graphene layers epitaxially grown on $\mathrm{SiC}^{25,26}$

To understand this behavior, it is useful to explore the asymptotic behaviors of graphene conductivities in the high doping limit. In highly doped graphene at lower energies, the interband transitions can be ignored and only the $E_{n} \rightarrow E_{n+1}$ $\left(E_{n}<\mu_{c}<E_{n+1}\right)$ intraband transition dominates in Eqs. (2a) and (2b). This leads to

$$
\begin{aligned}
\sigma_{x x}(\omega) & =\frac{e^{2}}{2 \pi \hbar} \frac{i \omega_{B}\left(\omega+i \tau^{-1}\right) f_{\text {intra }}^{-1}(n)}{\left(\omega+i \tau^{-1}\right)^{2}-f_{\text {intra }}^{2}(n) \omega_{B}^{2}}, \\
\sigma_{x y}(\omega) & =\frac{e^{2}}{2 \pi \hbar} \frac{\omega_{B}^{2}}{\left(\omega+i \tau^{-1}\right)^{2}-f_{\text {intra }}^{2}(n) \omega_{B}^{2}} .
\end{aligned}
$$

Applying $E_{n}=\sqrt{n} \hbar \omega_{B}=\mu_{c}$ and the approximation

$$
f_{\text {intra }}(n)=\frac{1}{\sqrt{n+1}+\sqrt{n}} \approx \frac{1}{2 \sqrt{n}} \approx \frac{\hbar \omega_{B}}{2 \mu_{c}}
$$


yields

$$
\begin{aligned}
\sigma_{x x}(\omega) & =\frac{e^{2} \mu_{c}}{\pi \hbar^{2}} \frac{i\left(\omega+i \tau^{-1}\right)}{\left(\omega+i \tau^{-1}\right)^{2}-\omega_{c}^{2}}, \\
\sigma_{x y}(\omega) & =\frac{e^{2} \mu_{c}}{\pi \hbar^{2}} \frac{\omega_{c}}{\left(\omega+i \tau^{-1}\right)^{2}-\omega_{c}^{2}},
\end{aligned}
$$

where $\omega_{c}=e B v_{F}^{2} / \mu_{c}$, in analogy to classical cyclotron frequency by introducing $m^{*}=\mu_{c} / v_{F}^{2}$. Equations (15a) and (15b) behave like a conventional 2DES. Although the cyclotron frequencies in graphene are $\propto \sqrt{B}$ [see Eq. (1)], highly doped graphene will exhibit classical cyclotron resonances, ${ }^{35} \omega_{c} \propto B$. For these classical Drude-type magneto-optical conductivities, the spectrum of optical active $(l=1)$ magnetoplasmon excitations in the dot has the form ${ }^{36,37}$

$$
\omega_{ \pm}=\sqrt{\omega_{p}^{2}+\omega_{c}^{2} / 4} \pm \omega_{c} / 2 .
$$

We can obtain $\Delta \omega_{ \pm} \approx \omega_{c} / 2$ in our situations, where $\omega_{p} \gg \omega_{c}$. This is also in agreement with the results above, e.g., $\Delta \omega_{ \pm}=0.018 \omega_{R}$ shown in Fig. $1(\mathrm{~b})$, where according to the definition, $\omega_{R}=0.17 \mathrm{eV}$ and $\omega_{c}=6.25 \mathrm{meV}$, leading to $\Delta \omega_{ \pm}=0.49 \omega_{c}$.

\section{CONCLUSIONS}

In this article, we have studied the edge magnetoplasmons in graphene disks and the corresponding optical excitations. In the absence of magnetic fields, we found the dipole plasmon energy approximately given by the $2 \mathrm{D}$ plasmon energy at wavelength $\lambda=2 \pi R$. In the presence of magnetic fields, at $l \neq 0$ two magnetoplasmons assigned to different edge current rotations can be excited separately by using circularly polarized waves, and their spectra are oppositely shifted and the shifted energies depend linearly on the magnetic field, e.g., $\Delta \omega_{ \pm} \approx \pm \omega_{c} / 2$ for the $l=1$ mode. This phenomenon can be understood by considering the classical magneto-optical conductivities in the high doping limit.

\section{ACKNOWLEDGMENTS}

This work was funded by the Swedish Research Council (VR). *weihua.wang@chalmers.se

${ }^{1}$ Z. Fei, G. O. Andreev, W. Bao, L. M. Zhang, A. S. McLeod, C. Wang, M. K. Stewart, Z. Zhao, G. Dominguez, M. Thiemens, M. M. Fogler, M. J. Tauber, A. H. Castro-Neto, C. N. Lau, F. Keilmann, and D. N. Basov, Nano Lett. 11, 4701 (2011).

${ }^{2}$ A. K. Geim, Science 324, 1530 (2009).

${ }^{3}$ K. F. Mak, M. Y. Sfeir, Y. Wu, C. H. Lui, J. A. Misewich, and T. F. Heinz, Phys. Rev. Lett. 101, 196405 (2008).

${ }^{4}$ C.-F. Chen, C.-H. Park, B. W. Boudouris, J. Horng, B. Geng, C. Girit, A. Zettl, M. F. Crommie, R. A. Segalman, S. G. Louie, and F. Wang, Nature (London) 471, 617 (2011).

${ }^{5}$ F. Wang, Y. Zhang, C. Tian, C. Girit, A. Zettl, M. Crommie, and Y. R. Shen, Science 320, 206 (2008).

${ }^{6}$ A. Vakil and N. Engheta, Science 332, 1291 (2011).

${ }^{7}$ P.-Y. Chen and A. Alú, ACS Nano 5, 5855 (2011).

${ }^{8}$ A. Y. Nikitin, F. Guinea, F. J. Garcia-Vidal, and L. Martin-Moreno, Phys. Rev. B 84, 195446 (2011).

${ }^{9}$ F. H. L. Koppens, D. E. Chang, and F. J. García de Abajo, Nano Lett. 11, 3370 (2011).

${ }^{10}$ J. Christensen, A. Manjavacas, S. Thongrattanasiri, F. H. L. Koppens, and F. J. García de Abajo, ACS Nano 6, 431 (2011).

${ }^{11}$ S. Thongrattanasiri, F. H. L. Koppens, and F. J. García de Abajo, Phys. Rev. Lett. 108, 047401 (2012).

${ }^{12}$ A. Y. Nikitin, F. Guinea, F. J. Garcia-Vidal, and L. Martin-Moreno, Phys. Rev. B 85, 081405 (2012).

${ }^{13} \mathrm{~S}$. Thongrattanasiri, A. Manjavacas, and F. J. García de Abajo, ACS Nano 6, 1766 (2012).

${ }^{14}$ W. Zhou, J. Lee, J. Nanda, S. T. Pantelides, S. J. Pennycook, and J.-C. Idrobo, Nat. Nanotech. 7, 161 (2012).

${ }^{15}$ E. Ozbay, Science 311, 189 (2006).

${ }^{16}$ F. Bonaccorso, Z. Sun, T. Hasan, and A. Ferrari, Nat. Photon. 4, 611 (2010).
${ }^{17}$ J. Chen, M. Badioli, P. Alonso-González, S. Thongrattanasiri, F. Huth, J. Osmond, M. Spasenović, A. Centeno, A. Pesquera, P. Godignon, A. Z. Elorza, N. Camara, F. J. García de Abajo, R. Hillenbrand, and F. H. L. Koppens, Nature (London) 487, 77 (2012).

${ }^{18}$ Z. Fei, A. S. Rodin, G. O. Andreev, W. Bao, A. S. McLeod, M. Wagner, L. M. Zhang, Z. Zhao, M. Thiemens, G. Dominguez, M. M. Fogler, A. H. Castro Neto, C. N. Lau, F. Keilmann, and D. N. Basov, Nature (London) 487, 82 (2012).

${ }^{19}$ O. L. Berman, G. Gumbs, and Y. E. Lozovik, Phys. Rev. B 78, 085401 (2008).

${ }^{20}$ R. Roldàn, J.-N. Fuchs, and M. O. Goerbig, Phys. Rev. B 80, 085408 (2009).

${ }^{21}$ A. M. Fischer, A. B. Dzyubenko, and R. A. Römer, Phys. Rev. B 80, 165410 (2009).

${ }^{22}$ J.-Y. Wu, S.-C. Chen, O. Roslyak, G. Gumbs, and M.-F. Lin, ACS Nano 5, 1026 (2011).

${ }^{23}$ O. G. Balev, P. Vasilopoulos, and H. O. Frota, Phys. Rev. B 84, 245406 (2011)

${ }^{24}$ W. Wang, J. M. Kinaret, and S. P. Apell, Phys. Rev. B 85, 235444 (2012).

${ }^{25}$ I. Crassee, M. Orlita, M. Potemski, A. L. Walter, M. Ostler, Th. Seyller, I. Gaponenko, J. Chen, and A. B. Kuzmenko, Nano Lett. 12, 2470 (2012).

${ }^{26}$ H. Yan, Z. Li, X. Li, W. Zhu, P. Avouris, and F. Xia, Nano Lett. 12, 3766 (2012).

${ }^{27}$ V. P. Gusynin, S. G. Sharapov, and J. P. Carbotte, J. Phys.: Condens. Matter 19, 026222 (2007).

${ }^{28}$ A. L. Fetter, Phys. Rev. B 33, 5221 (1986).

${ }^{29}$ W. Wang, P. Apell, and J. Kinaret, Phys. Rev. B 84, 085423 (2011).

${ }^{30}$ E. H. Hwang and S. Das Sarma, Phys. Rev. B 75, 205418 (2007).

${ }^{31}$ M. Jablan, H. Buljan, and M. Soljačić, Phys. Rev. B 80, 245435 (2009). 
${ }^{32}$ H. Pfnür, T. Langer, J. Baringhaus, and C. Tegenkamp, J. Phys.: Condens. Matter 23, 112204 (2011).

${ }^{33}$ T. Langer, D. F. Förster, C. Busse, T. Michely, T. Pfnür, and C. Tegenkamp, New J. Phys. 13, 053006 (2011).

${ }^{34}$ V. A. Volkov and S. A. Mikhailov, Sov. Phys. JETP 67, 1639 (1988).
${ }^{35}$ A. M. Witowski, M. Orlita, R. Stpniewski, A. Wysmołek, J. M. Baranowski, W. Strupiński, C. Faugeras, G. Martinez, and M. Potemski, Phys. Rev. B 82, 165305 (2010).

${ }^{36}$ S. J. Allen, H. L. Störmer, and J. C. M. Hwang, Phys. Rev. B 28, 4875 (1983).

${ }^{37}$ R. P. Leavitt and J. W. Little, Phys. Rev. B 34, 2450 (1986). 